\title{
Integration of Predictive Display and Aircraft Flight Control System
}

\author{
A.V. Efremov ${ }^{1, *}$, M.S. Tjaglik ${ }^{1}, I . H$. Irgaleev $^{1}$ and $V . G$. Tsipenko $^{2}$ \\ ${ }^{1}$ Moscow Aviation Institute, Aeronautical school, Moscow, Russia \\ ${ }^{2}$ Moscow State Technical University of Civil Aviation, Moscow, Russia
}

\begin{abstract}
The synthesis of predictive display information and direct lift control system are considered for the path control tracking tasks (in particular landing task). The both solutions are based on pilot-vehicle system analysis and requirements to provide the highest accuracy and lowest pilot workload. The investigation was carried out for cases with and without time delay in aircraft dynamics. The efficiency of the both ways for the flying qualities improvement and their integration is tested by ground based simulation.
\end{abstract}

\section{Introduction}

The improvement of flying qualities might be realized by the different ways. One of them is the usage of direct lift control (DLC) system providing the decoupling of path and angular motions. It allows to decrease the pole order in the origin in aircraft path motion dynamics $[1,2]$ and to get the slope of its amplitude frequency response characteristics close to $-20 \mathrm{~dB} / \mathrm{dec}$ in crossover frequency range. The other way is the usage of predictive display [3]. It influences on zeros of controlled element dynamics transfer function and allows to get the same slope of its amplitude frequency characteristic $(-20 \mathrm{~dB} / \mathrm{dec})$ in the same frequency range. The high potentiality of predictive display was investigated in flight tests [4] and in ground-based simulation [5]. In current paper it is considered two approaches to the design of predictive display differed by the way of determining the path angle. The effectiveness of the predictive display compares with effectiveness of DLC system. The last one was realized by two ways: by use command filters and by use the feedback and feed forward controllers. The additional requirement to the dynamics of DLC aircraft based on requirement of closeness of its frequency response characteristic to the optimal control dynamics [6] is considered. The efficiency of such additional requirement was checked in ground-based simulation. The potentialities of integration of DLC system and predictive display was investigated in ground based simulation.

\section{The usage of direct lift control (DLC) system}

A number of studies were dedicated to the usage of DLC system for flying qualities improvement $[1,2,7,8]$ where the requirement to execution of a specific mode of motion was formulated.

In the current investigation two versions of DLC system providing the variable angle of attack and constant pitch angle are considered. These versions are shown on fig 1-2. The version given on fig 1 provides the required mode of motion with help of command filters $W_{1}$ and $W_{2}$. The second version is based on the usage of the command filter in the angle of attack loop and feed forward controller in the other loop filter fig. 2 . Two actuator dynamics with and without rate limit were investigated.

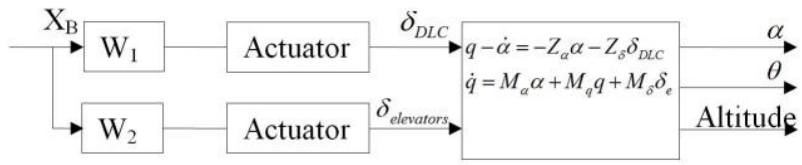

Fig 1. DLC system with command filters

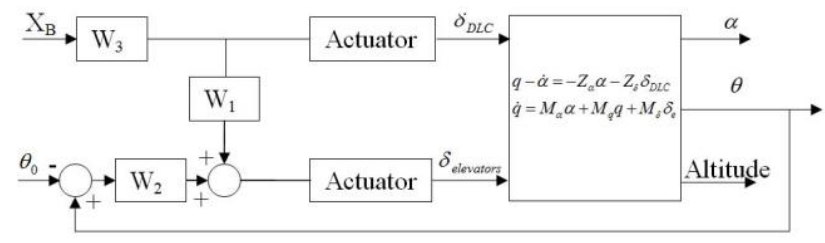

Fig 2. DLC system with command filters and feedback and feed forward controllers

The equations for the filters and controllers were defined by using the equations of short period motion. For any versions of the considered DLC systems the use of DLC leads to the decrease of pole order in the origin of the controlled element dynamics (table 1). It provides the simplification of pilot behavior (the decrease of his

Corresponding author: pvl@mai.ru 
lead compensation). As a consequence, the accuracy in tracking task has to be improved too.

Preliminary experiments were executed on the MAI workstation where the operator carried out the single loop tracking task with the input signal $i(t)$ characterized by the low bandwidth spectrum $S_{i i}=K^{2} /\left(\omega^{2}+\omega_{i}^{2}\right)^{2}$, where $\omega_{i}=0.2 \mathrm{rad} / \mathrm{sec}$.

Table 1. $\mathrm{W}_{\mathrm{C}}$ for different versions of DLC system

\begin{tabular}{|c|c|c|}
\hline & version 1 & version 2 \\
\hline $\mathrm{W} 1$ & $K_{C}$ & $\frac{W_{3} \cdot\left(-Z_{\delta}\right) \cdot M_{\alpha}}{M_{\delta} \cdot\left(p-Z_{\alpha}\right)}$ \\
\hline $\mathrm{W} 2$ & $\frac{W_{1} \cdot\left(-Z_{\delta}\right) \cdot M_{\alpha}}{M_{\delta} \cdot\left(p-Z_{\alpha}\right)}$ & $K_{C}$ \\
\hline $\mathrm{W} 3$ & --- & $K_{C}$ \\
\hline $\mathrm{Wc}=\frac{H}{X_{e}}$ & $\frac{V \cdot\left(-Z_{\delta}\right)}{p \cdot\left(p-Z_{\alpha}\right)}$ & $\frac{V \cdot\left(-Z_{\delta}\right)}{p \cdot\left(p-Z_{\alpha}\right)}$ \\
\hline
\end{tabular}

The measurement of the accuracy demonstrated that the close results were achieved for two versions of DLC system (fig3). In case of «ideal» actuator (when the rate limit was not tacken into account) the variance of error was three times higher in comparison with experiments carried out for aircraft without DLC system.

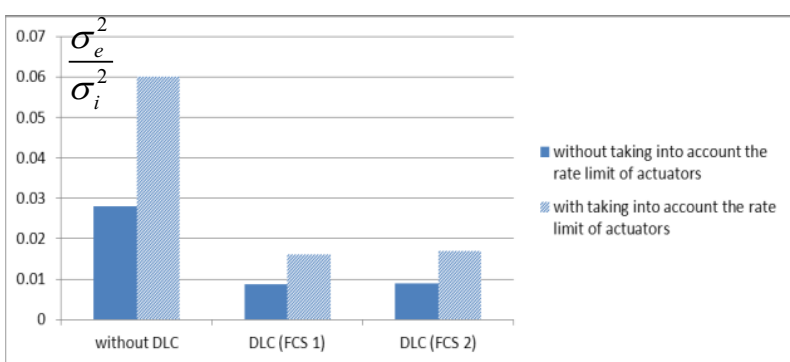

Fig 3. Influence of DLC on accuracy

Except it the measurements of pilot describing function demonstrated that pilot lead compensation was considerably lower (fig 4).

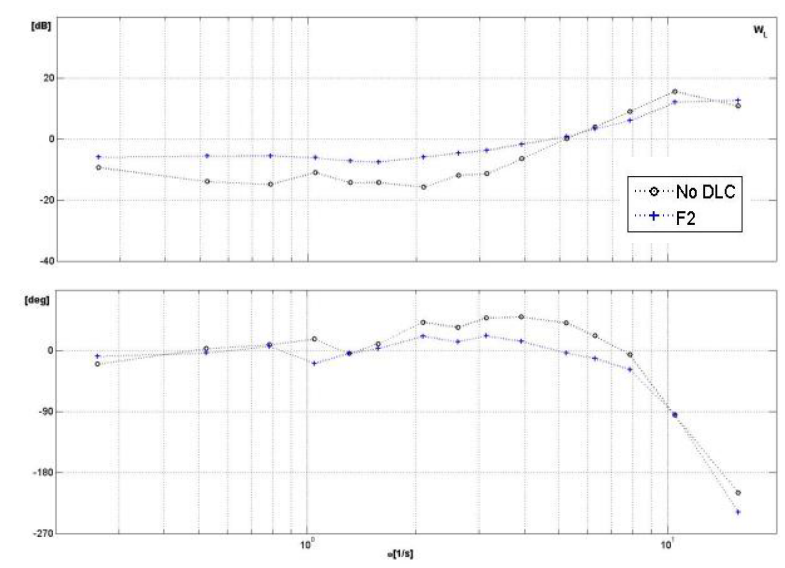

Fig 4. Pilot frequency response characteristics for aircraft with and without DLC
In case when the rate limit was taken into account in the model of actuator dynamics, the variance of error was higher although the effect of DLC increased up to 44.5 times. Because the parameters of filters are defined by the aerodynamic coefficients (see table 1), their inaccurate knowledge does not allow to keep the required mode of motion. The evaluation of influence of such inaccurcy on the required mode of motion was examined on simulator. The experiments demonstrated that inaccuracy of the knowledge of coefficients $\left(M_{\alpha}, Z_{\alpha}, Z_{\delta}\right)$ up to $30 \%$ didn't have any practical influence on variance of error.

Except the requirement to the mode of motion, an additional one - the closeness of DLC aircraft dynamic to the optimal controlled element dynamics ( $\left.W_{C}^{\text {opt }}\right)$, was added.

The optimal control dynamics was defined in [6,9] as the transfer function provided the highest accuracy $\sigma_{e}^{2}=\sigma_{\text {emin }}^{2}$ with the simplest type of pilot behavior when his describing function corresponds to $W_{P}(j w)=K_{P} e^{-j w \tau}$, and spectral density of pilot noise $n_{e}(t)$ ("remnant") $S_{n_{e} n_{e}}=K_{n_{e}} \sigma_{e \min }^{2}, \quad K_{n_{e}}=0,01$. The Wiener approach was used for the evaluation with taking into account the fixed part - non minimum phase element $e^{-s \tau}$. According to $[6,9]$, the general equation for the $W_{C}^{\text {opt }}$ obtained by use of Wiener approach is the following:

$$
W_{C}^{\text {opt }}(s)=\frac{M_{0}(s) \hat{P}^{-}(s)}{A^{+}(s) \hat{P}^{-}(s)-M_{0}(s) P^{-}(s)},
$$

where $P^{-}(s)$ and $\hat{P}^{-}(s)$ are the nominator and denominator of the time delay Pade approximation.

$$
W_{O}(s)=e^{-s \tau} \cong \frac{2-s \tau}{2+s \tau}=\frac{P^{-}(s)}{\hat{P}^{-}(s)} .
$$

The polynomials $A^{*}(s)$ and $C(s)$ can be defined by factorization of equation for the input signal spectral density:

$$
S_{i i}(s)=\frac{C(s)}{D(s)}=\frac{C^{+}(s) C^{-}(s)}{D^{+}(s) D^{-}(s)}
$$

and spectral density:

$$
S(s)=S_{i i}(s)+S_{n_{e} n_{e}}(s)=\frac{A(s)}{B(s)}=\frac{A^{+}(s) A^{-}(s)}{B^{+}(s) B^{-}(s)} .
$$

The polynomial $M_{0}(s)$ is defined by equation

$$
P^{-}(s) M_{0}(s) A^{-}(s)+B^{+}(s) L_{0}(s)=C(s) \hat{P}^{-}(s) .
$$

With taking into account the remnant spectral density model given above it is possible to get that $B(s)=D(s)$ and equation for $W_{C}^{\text {opt }}(s)$ :

$$
W_{C}^{\text {opt }}(s)=\frac{A^{-}(s) M_{0}(s) \hat{P}^{-}(s)}{D^{+}(s)\left[N^{2} D^{-}(s) \hat{P}^{-}(s)+L_{0}(s)\right]},
$$

where $N^{2}=S_{n_{e} n_{e}}$.

It is shown in [8] that 


$$
N^{2}=S_{n_{e} n_{e}}=\frac{\sigma_{e m i n}^{2}}{\frac{1}{K_{n_{e}}}-\int_{0}^{\infty}\left|W_{C L}\right|^{2} d \omega} .
$$

For the case when $S_{i i}=\frac{K^{2}}{\left(\omega^{2}+\omega_{i}^{2}\right)^{2}}$ :

$$
\begin{aligned}
& A^{+}(s)=\sqrt{K_{n_{e}} \sigma_{e m i n}^{2}\left(s^{2}+b s+c\right)} ; \\
& A^{-}(s)=\sqrt{K_{n_{e}} \sigma_{e m i n}^{2}\left(s^{2}-b s+c\right)} ;
\end{aligned}
$$

where $\quad b=\sqrt{2\left(\omega_{i}^{2}+c\right)}, \quad c=\sqrt{K_{i}^{2}} /\left(K_{n_{e}} \sigma_{\text {emin }}^{2}\right)+\omega_{i}^{4}$, $B^{+}(s)=\left(\omega_{i}+s\right)^{2}, C_{p}=K_{i}^{2}$.

These equations allows transforming (1) to the following:

$$
\begin{gathered}
(2-s \tau) M_{0}(s) \sqrt{K_{n_{e}} \sigma_{\text {emin }}^{2}}\left(s^{2}-b s+c\right)+ \\
+\left(\omega_{i}+s\right)^{2} L_{0}(s)=K_{i}^{2}(2+s \tau)
\end{gathered}
$$

The solution of this equation is the following:

$$
M_{0}(s)=a_{0}+b_{0} s, L_{0}(s)=c_{0}+d_{0} s+f_{0} s^{2}
$$

The coefficients $a_{0}, b_{0}, c_{0}, d_{0}, f_{0}$ can be defined by solution of the system of algebraic equations of the 5 order which can be obtained by the equate coefficients of the equation (2) with the same order of 's'.

For the case where $K_{n_{e}}=0,01$; $\tau=0,15 \mathrm{sec} ; \omega_{i}=0,2 \mathrm{rad} / \mathrm{sec}$ the frequency response characteristics of the aircraft optimal dynamics is shown on fig 5 .

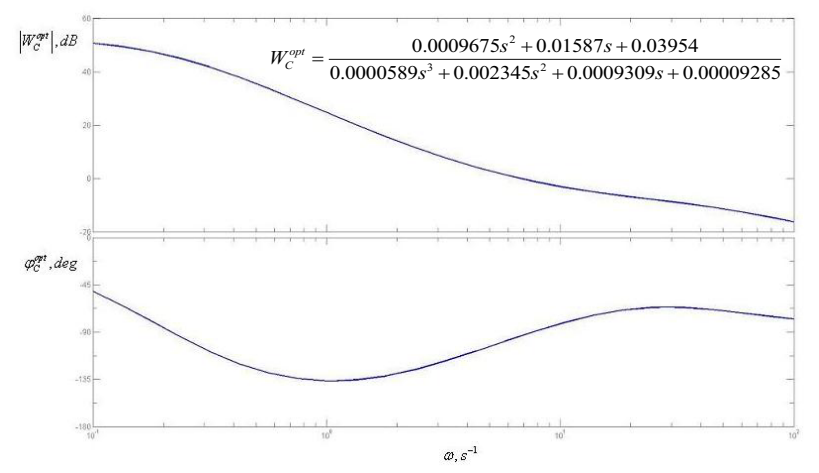

Fig 5. Frequency response of optimal control dynamics

It was offered to include the filter $W_{f}$ in flight control system for the improvement of the accuracy. Parameters of the filters are selected from the requirement of closeness of controlled element dynamics to the optimal one $W_{C}^{\text {opt }}$. The controlled element dynamics are the dynamics of aircraft with DLC system providing the mode of motion $-\Delta \alpha=v a r, \Delta \theta=0$, and additional filter. The general equation for this filter is the following $W_{f}=K_{1} \frac{T_{1} s+1}{T_{2} s+1}$. The selection of the filter parameters was carried out by minimization of criteria:

$$
\mathrm{I}=\min _{T_{1}, T_{2}} \sum_{w_{i}}\left[|| W_{C}|-| W_{C}^{\text {opt }} \|^{2}+\alpha\left(\varphi_{c}-\varphi_{\text {copt }}\right)^{2}\right]
$$

where $\left|W_{C}\right|$ and $\left|W_{C}^{\text {opt }}\right|$ - amplitude frequency response characteristics of controlled element dynamics and aircraft optimal dynamics, $\varphi_{c}$ and $\varphi_{c \text { opt }}-$ phase frequency response characteristics of the same dynamics, $\alpha$ - weighting coefficient value equal to $\frac{1}{57,3}$. The frequencies $\omega_{i}$ are the frequencies of polyharmonic input signal $i(t)=\sum_{k} A_{k} \cos \left(\omega_{k} t\right)$ widely used in MAI researches [5, 9] for pilot-aircraft system analysis. The values of the filter parameters are given in the table 2 for the case of actuator with ideal dynamics. For the both versions of DLC system these paramiters are the same $\left(\mathrm{K}_{1}=1.46, \mathrm{~T}_{1}=0.34, \mathrm{~T}_{2}=0.1\right)$ and the actuator dynamics does not influence on these values.

Experiments executed on the workstation demonstrated that installation of additional filter $W_{f}$ allowed to decrease the variance of error in 1,6-1,7 times (fig 6). For the case when the rate limit was taken into account in the actuator dynamic the effectiveness of the additional filter is higher in 1,5 times.

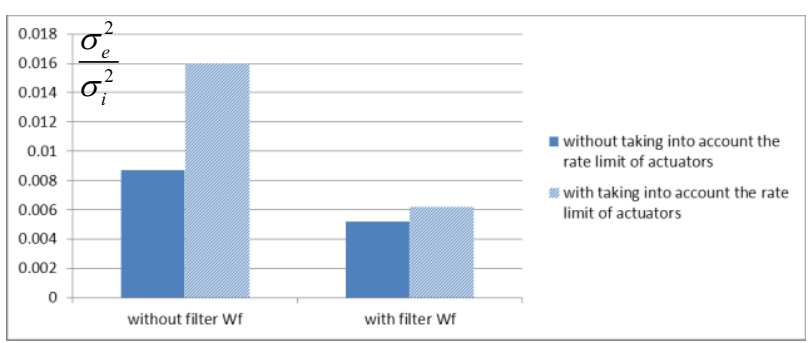

Fig 6. Influence of additional filter

\section{The usage of predictive display and its integration with DLC}

Two versions of predictive information were considered. The first one (1) (fig 7) supposes that the on-board computer generates the predictive vector of the path angle $\gamma_{p r}=\gamma+\dot{\gamma} T / 2$.

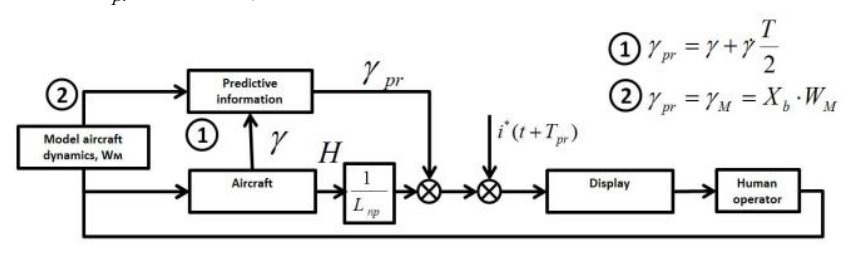

Fig. 7. Pilot-vehicle system with predictive displays

Here the path angle $\gamma$ is estimated by the inertial system and $\dot{\gamma}$ is the calculated derivative of the path angle. Through the display pilot perceives the predictive aiming angle $\quad \varepsilon_{p r}=\Delta H / L+\gamma_{p r}$, where $L=V T_{p r} \quad\left(T_{p r}-\right.$ predictive time, $V$ - aircraft velocity) is the distance between the pilot's eye and the surface perpendicular to the $3 \mathrm{D}$ corridor generating on the display and limiting the space where the aircraft, has to move. 
The other version of predictive information (fig 7) supposes that the on-board computer calculates the path angle $\gamma_{m}$ according to the mathematical model ( $W_{M}$, fig 7,2) of the aircraft motion $W_{M}$. In that case the pilot perceives the angle $\varepsilon_{p r}=\Delta H / L+\gamma_{p r}$ on the screen of display. Here $\gamma_{m}=X_{e} W_{M}$.

In case when the time delay is accompanied the control process the transfer function of the controlled element dynamics is equal to

$$
\frac{\varepsilon}{X_{e}}=\frac{K_{c}\left(T_{n p} s^{2}+2 s+\frac{2}{T_{n p}}\right)}{2 s^{2}\left(s^{2}+2 \xi \omega s+\omega^{2}\right)} e^{-\tau s} .
$$

For the second version of predictive display $W_{M}$ does not include time delay. The frequency response characteristics for these two versions are shown on. fig. 8 for $\tau=0.18$. It is seen that in case when time delay exist in dynamics the second version allows to suppress its effect.

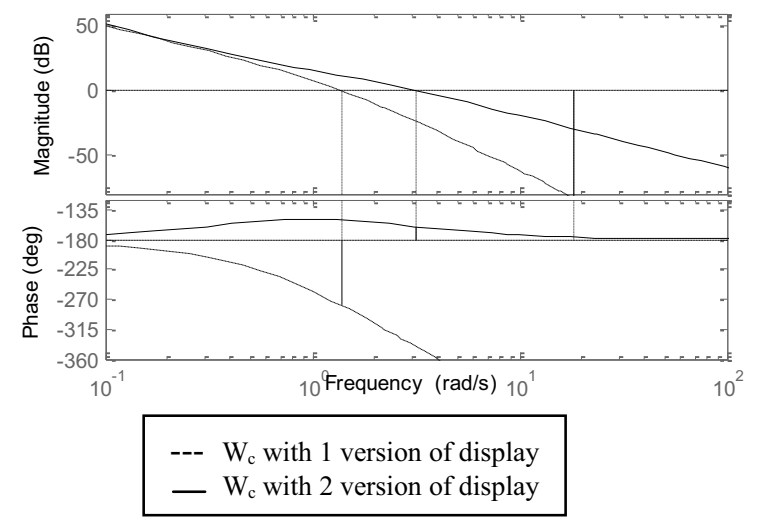

Fig 8. Influence of predictive display on $W_{C}(j w)$

The determination of predictive display efficiency requires the preliminary definition of predictive time $T_{p r}$ (or $L_{p r}=T_{p r} V$ ). For that purpose it was developed the procedure based on the modeling of the pilot-vehicle system shown on fig 9.

Here $i(t)$ is the program trajectory and $i(t+\tau)$ is the predictive trajectory projected on display. The optimal $T_{p r}\left(L_{p r}\right)$ was defined as the value corresponding to the minimum variance $\sigma_{\Delta H}^{2}$, where $\Delta H=H-i(t)$. For the determination of $T_{p r_{o p t}}$ it was carried out the mathematical modeling of the single loop pilot-vehicle system for each value $T_{p r}$.

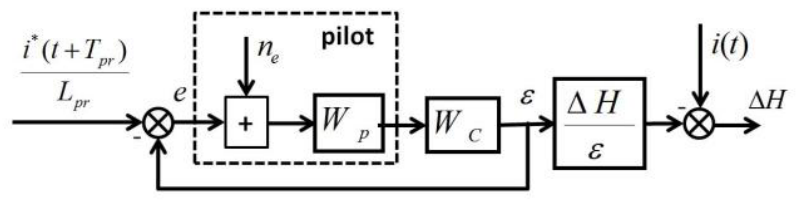

Fig 9. Pilot aircraft system for calculation $\sigma_{\Delta_{H}}^{2}$
For that purpose it was used the pilot structural model [5]. The result of such mathematical modeling are the pilot model parameters $\left(K_{p}, T_{L}, \ldots\right)$ used then for calculation of variance of $\sigma_{\Delta_{H}}^{2}$ and for definition of its minimum (fig. 10).

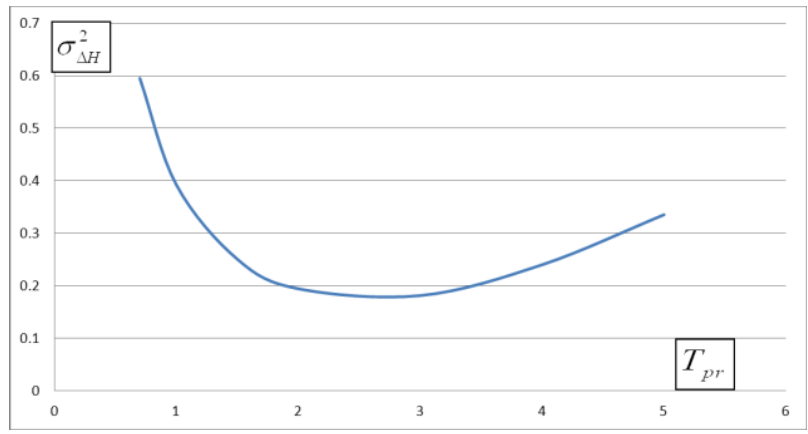

Fig 10. Variance of $\sigma_{\Delta H}^{2}=f\left(T_{p r}\right)$

The efficiency of two predictive display versions were studied on the specialized simulator (fig 11). The piloting task was the landing task where the pilot had to track the glide slope and to compensate the error signal $\varepsilon_{p r}$.

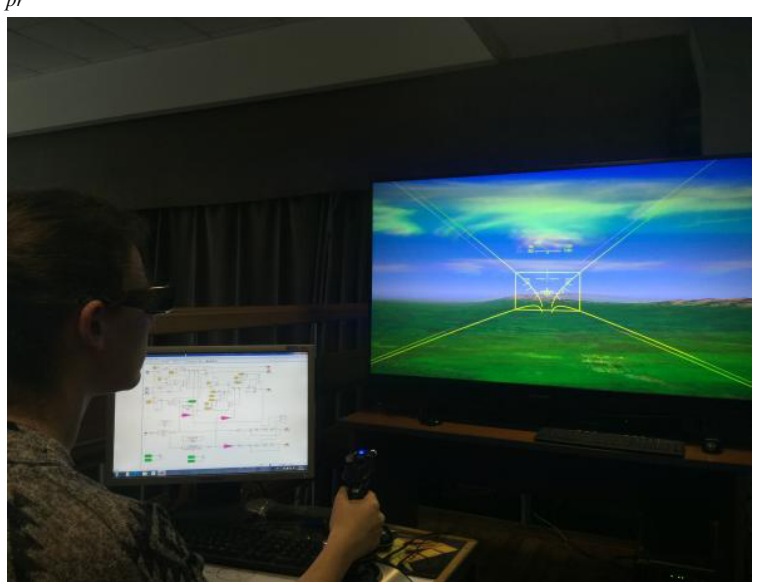

Fig 11. Specialized simulator

The results of experiments executed without the disturbances demonstrated that the both versions of predictive display provide approximately the same accuracy in glide slope tracking (fig 12, case $\tau=0$ ).

In case when $\tau$ was not zero $(\tau=0.18)$ the usage of the second version of display allowed to suppress the effect of controlled element dynamics and to achieve higher accuracy in 1.7 times.

The simultaneous usage of DLC system and predictive display improves the accuracy on about 10$15 \%$. In case when the disturbances were not zero the effect of integration DLC system and predictive display increases up to $40 \%$.

In particular all these results can be explain with help of fig 13. There are given the values of criteria I (3) for the different cases studied in this research. This criteria defines the difference between the amplitudes and phases of the alternative dynamics and optimal frequency response characteristics. 


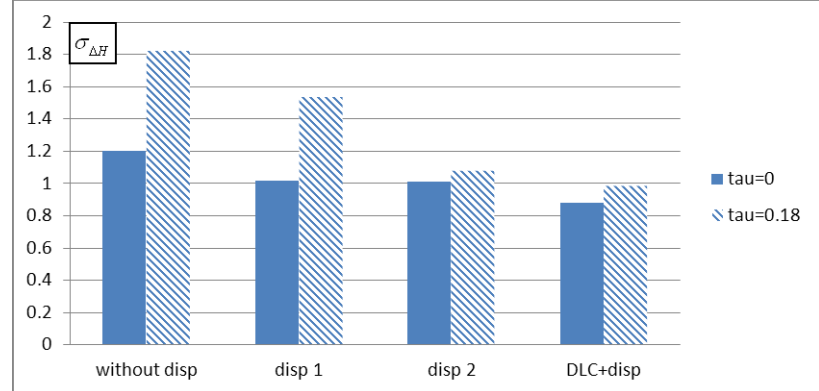

Fig. 12. The variances of error for the different means of augmentation (disp 1 - first version of predictive display, disp $2-$ second version of predictive display)

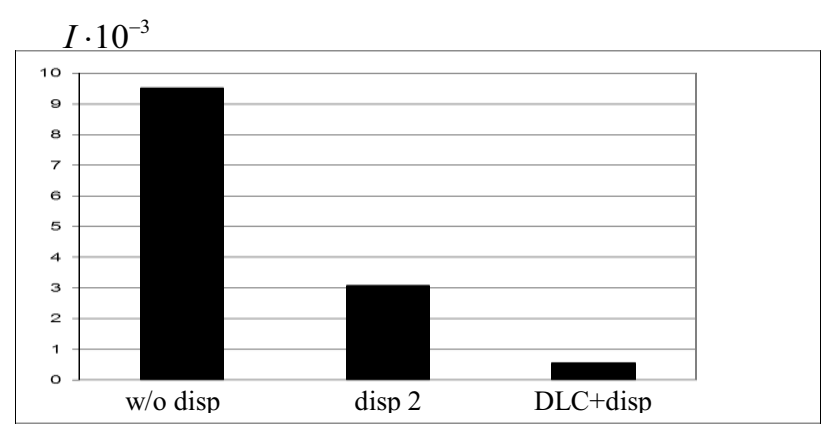

Fig.13. Variances of criteria I (see equation (3))

It is seen that the integration of the DLC system and predictive display provides the highest closeness of the controlled element dynamics to the optimal one.

The results included in the paper are the results of the investigation sponsored by the Ministry of Education and Science (contract № 9.1081.2014/K).

\section{References}

1. J.P. Guskov, G.I. Zagainov, Aircraft Flight Control (Moscow, Machinostroeniye, 1980)

2. A.V. Efremov, A.V. Ogloblin, The Ways for Provision of Necessary Flying Qualities. In the book "The Problem of Flight Dynamics of Aircraft Provided by DLC" (Moscow, MAI, 1981).

3. A.V. Efremov et al., Journal Russian Aeronautics 2 (2016)

4. G. Sachs, Journal of Guidance, Control, and Dynamics, 23(3) (2000)

5. A.V. Efremov, M.S. Tjaglick, The Development of Perspective Displays for Highly Precise Tracking Tasks. In the book "Advances in Aerospace Guidance, Navigation and Control" (Springer, 2011).

6. A.V. Efremov et al., Pilot as a Dynamic System. (Moscow, Machinostroeniye, 1993)

7. J.P. Guskov, Analysis of Requirements to the Aircraft Handling Qualities Provided by DLC in the Stabilization Task. In the book "The Problem of Flight Dynamics of Aircraft Provided by DLC”, (Moscow, MAI, 1981)

8. R. Hoh, J. Ashkenas, et al. Development of Handling Qualities Criteria for Aircraft with Independent Control of Six Degrees of Freedom. AFWAL-TR-80 (1980).

9. A.V. Efremov et al. Investigation of Pilot Induced Oscillation Tendency and Prediction Criteria Development. WL-TR-96-3109 (Wright Lab USA, 1996). 